any of the intra-abdominal operations on the round ligaments, and I have seen no instance of failure, even after three subsequent pregnancies. As an indication of the limited number of cases suitable for the Alexander-Adams operation, I would say that in a large yearly operative turn-over in hospital and private, perhaps about twelve cases will be found where the operation can be really recommended. Peradventure even twelve successful cases per annum in the experience of one operator may lead Sir William Smyly and Dr. Bethel Solomons to withhold their threatened death-blow from the Alexander-Adams operation.-I am, etc.

$$
\text { James Haig Ferguson, M.D., }
$$

Elinburgh, Nov. 26th. F.R.C.S.Edin.

\section{TREATMENT OF FEVER.}

Sir,-I have read with interest the letter in your issue of November 18th (p. 1000) containing arguments in favour of treating various acute diseases with a compound powder containing aspirin gr. $\mathrm{x}$, phenacetin gr. $\mathrm{v}$, and pulv. ipecac. co. gr. $\mathbf{v}$.

My experience with this combination of drugs in acute specific diseases and in all conditions of acute toxaemia has been very similar to that described by Surgeon Commander Parnell and others. In my opinion the importance of this line of treatment cannot be overestimated.

As I understand it, recovery from acute toxaemia (any acute disease) is due to that increase of resisting power which we call "acquired inmunity," and that the essence of such acquired immunity is an habituation to the particular toxin of the disease from which the patient recovers-just as a man becomes used to nicotine or a horse to diphtheria toxin.

There are three ways in which an individual may become habituated to a toxin: (1) by a huge and continuous dose, as in disease-a dangerous method; (2) by weakening the toxin and so providing stepping stones for reaction, as in vaccina tion and serum treatment; and (3) by giving small doses of the toxin, as in tobacco and opium smoking and in those cases in which individuals remain immune to a disease (for example, yellow fever) as long as they dwell in a country where it is prevalent but acquire it after return from a country where it is not prevalent.

This new treatment applies the third method. By means of it toxins are continually withdrawn in the sweat, so that the individual experiences a series of small doses. The applications of the treatment are more nume: ous, and it is more easily applied than the serum treatment. At any rate it opens out a new line of inquiry and ultimately may prove as useful as the serum treatment itself. In conjunction with serum treatment (for example, in diphtheria) it is aamirable.

Doubtless there are injurious ways of lowering the temperature, and it is an axiom in medicine that the temperature in acute diseases should not be too much lowered by artificial means; but only those who have employed this treatment when themselves suffering from acute disease are able to appreciate the extraordinary and immediate relief and benefit which follows. What can be the explanation other than that which I have indicated?-I am, etc.,

W. Bastian, Surgeon Commander R.N.

H.M.S. Victory, Portsmouth, Nov. 20th.

Sir,-The prescription given in the letter on the treatment of fever in your issue of November 18th (p. 1000) was extensively used in the British Stationary Huspital, Baghdad.

It was used as an almost universal prescription in most of the fever cases admitted. Admissions numbered anything up to 35 per diem during the sandfly period of the year, and it invariably relieved the symptoms. 'l'o the first powder given on admission calomel gi. iv was aclded.

During one busy day a sergeant (R.A.F.) was admitted with typical symptoms of sandfly fever. I noted, however, that the pain in the back was more severe than usual; the temperature on pain in the back was more severe than usual; the temperature on fever. The above prescription was used. The following day he cover. The above prescription was used. The following day he complained of heat rash. This struck me as peculiar, as his temperature was normal; on examining him 1 found him to be
suffering from small-pox. His symptoms were only mild, and he suffering from small-pox. His symptoms were only mild, and he was never very ill; he had never been vaccinated. This may have
been due to the elimination of toxins through the skin in the early period of the disease. As he was sent to the isolation hospital the case was lost sight of; unfortunately just one month later, when convalescence was just established, he died suddenly, from what cause I do not know.

We got to believe in this prescription to sucl an extent that we invariably gave it to all heatstroke cases. During the hot weather of 1921 the percentage of deaths from heatstroke was much lower than it had been previously; whether this had anything to do with the administration of this prescription is, of course, an open question, but it certainly tends to give one faith in using it.- I am, etc.,

London, W.C., Nov. 23rd.

\section{T. Gelston AtKins,}

Captain R.A.M.C.

SIR,-I noted with considerable interest the joint letter of Surgeon Commander Parnell, Dr. Green, and Sir Archdal Reid. For several years I have employed an almost identical prescription with such general satisfaction that I sometimes accuse myself of using it almost as a placebo. The intention is very remote from this, and although its original use was in some degree empirical it had a liberal element of pharmacological justification. The prescription is very simple Aspirin, pulv. ipecac. co. - āā gr. x. During the paroxysms of malaria its effect is extremely comforting and beneficial, as I demonstrated in a recont paper (Medical Officer, October 21st, 1922). In the prodromal stages of ordinary catarrhal colds and influenza it produces not only a sense of well-being but I am sure often stops further developments. In the notorious P.U.O. of active warfare days, which no doubt had a definite though unknown pathology, one found its use very satisfactory. In such cases as parotitis appearing as a metastatic effect following laparotomies I have seen marked relief. In short, in most febrile states, including the exanthemata, its effect is marked and valuable.

Whether its use is specific or purely palliative, as one is almost compelled to suppose, it is nevertheless a most useful simple combination. Pulv. ipecac. co. in doses of gr. $x$ practically never induces emesis in adults. $-\Upsilon \mathrm{am}$, etc.,

London, S.E., Nov. 18th. Donald Buchanan.

\section{THE SIIALL-POX OUTBREAK AT POPLAR} WORKHOUSE

SrR,-10r. C. S. Thomson, in his letter in the British Medical Jouknal for November 25 th, says that:

The "unmodified first case, in actual practice, would not be ikely to oblige by walking right into the arms of a medical man. More than likely the sufferer would have visited forty or fifty public houses, churches, or cinemas," etc.

I suggest that Dr. Thomson is quite wrong. The unmodified case of small-pox, in my experience, is much too ill to visit places of entertainment, and if he does not go to the doctor he goes home to bed and sends for the doctor to come to him.

Dr. 'Thomson tells us that his prescription for preventing mall-pox is "vaccination at infancy, revaccination at 12 to 14 , and a third session at 21," with additional vaccinations as and when necessary. I quite admit that if such a prescription could be applied to the whole population, without exception, small-pox would be quite effectually controlled; but $\mathrm{Dr}$. Thomson must know quite well that such a prescription never has been, is never likely to be, and indeed never could be applied in this or any other country. Moreover, granted that it could be applied, I venture to suggest that the premium to be paid (in the form of so much vaccination) is out of all proportion to the risk to be insured against. Why saturate forty-five millions of people, old and young, with vaccination when there is good reason to believe, from the experience of the past twenty years, that small-pox can be effectually con. trolled without it? As well advocate the repeated inoculation of the entire population of this country against enteric fever. Perhaps Dr. Thomson would do so, but few common-sense business men would think of listening to him.

As regards Dr. Stansfield's letter, he says that even if we got rid of the danger arising from modified small-pox in vaccinated subjects there would still remain a possibility of missed cases in naturally mild small-pox in unvaccinated persons. My answer to this is that naturally mild small-pox tends to "breed true," and gives rise to a mild strain of small. pox with a negligible mortality (small-pox of this type is pox wath that is often made about it). Small paccination, on the other hand, does not breed true, and, so far from giving rise to modified small-pox, it may and often does spread a very virulent form of the disease Hence vaccinated (but incompletely protected) disease. Hence vaccinated dar be a far greater danger to their neighbours than the unvaccinated, under present-day conditions, are ever likely to be.

It is interesting to learn that all of the 22 fatal cases in the present outbreak had been vaccinated, presumably in infancy. 